\title{
Gene therapy safety issues come to fore
}

Gene therapy is once again in the spotlightbrought there by news reports of "adverse events," including half a dozen deaths, involving participants in several separate clinical trials. Although none of these deaths has yet been attributed directly to the gene transfer procedures being evaluated, the mere possibility of such a connection is stirring intense interest, and highlights some of the complications that arise from dual oversight of the field.

Concerns over the unexplained deathsthe first for gene therapy-have officials at both the US Food and Drug Administration (FDA; Rockville, MD) and US National Institutes of Health (NIH; Bethesda, MD), who for nearly a decade have shared oversight responsibilities for gene therapy research, scrambling to make sense of those reports while also dealing with the clamor that they are causing.

The immediate focus is on the safety of vectors that are based on genetically modified versions of human adenovirus, several of which are being used to deliver genes into patients involved in a variety of clinical trials, including those in which the recent deaths occurred. Related concerns over use of a similar vector and clinical trial design led FDA officials in mid-October to halt temporarily two gene therapy clinical trials being run by Schering-Plough (Madison, $\mathrm{NJ}$ ), even though no gene therapy-related or unexplained deaths have been reported in either of those trials.

Promising to address these concerns in a "collegial" rather than an adversarial spirit, officials from NIH and FDA plan to devote a major part of the mid-December NIH Recombinant DNA Advisory Committee (NIHRAC) meeting to extensive deliberations over safety. "This is meant to be a positive meeting. . all about adenovirus vectors, including different constructs," says Amy Patterson, who directs the NIH Office of Recombinant DNA Activities (ORDA).

Participants at the December meeting also are being asked to consider a broad variety of issues related to "adenovirus vector safety and toxicity," including how such vectors are prepared, how they have been administered to subjects in clinical trials, and what kinds of illnesses those trial participants are being or have been treated for and what adverse events they may have experienced. "We have made efforts to contact all principal investigators using [such] vectors and asked them to present data. . .from a full range of [gene therapy] applications to see what lessons can be learned," says Patterson.

The first of the unexplained deaths, reported to the NIH and FDA in September, involves an 18-year-old man with a rare metabolic disorder that can cause dangerous build up of ammonia in the body. He was participating in a gene therapy clinical trial, directed by James Wilson at the University of Pennsylvania (UPenn, Philadelphia, PA), where an adenovirus vector was in use (Nature, 401, 517, 1999). NIHRAC meeting participants are to focus considerable attention on the death in the UPenn trial, in part because of its suddenness, because there is no obvious explanation for it, and also because Wilson and his colleagues were asked to assemble and analyze data from a number of recent studies examining the safety profile of adenovirus vector constructs in the wake of their patient's death.

\section{An important issue that has dogged both NIH and FDA is how to find an appropri- ate balance between public disclosure and confidential- ity when considering ongo- ing trials.}

Some of those preliminary data indicate that such vectors can cause serious problems when tested in certain animals.

Since early 1998, according to Patterson, at least six deaths have occurred in two other gene therapy trials involving patients with heart disease in which adenovirus vectors are being used. One of those trials is under the direction of Ronald Crystal at Cornell University Medical College (New York); the other is being led by Jeffrey Isner at Tufts University School of Medicine (Boston, MA). They have attributed those deaths to the patients' respective underlying diseases, rather than to the experimental gene therapy interventions being evaluated. Although information about these deaths apparently was reported promptly to the FDA, not all the details were shared immediately with NIH. This reporting discrepancy points to an important issue that has dogged both NIH and FDAhow to find an appropriate balance between public disclosure and confidentiality when considering ongoing trials.

The FDA and NIH share responsibilities for safeguarding gene therapy. FDA, which has regulatory authority and conducts nonpublic reviews, legally requires the reporting of all adverse events during trials. Presence of the NIH is considered valuable for providing additional expertise, a more public forum than a purely FDA-centered review could achieve, and a mechanism for reassuring the public about unusually sensitive safety and ethical issues associated with this research; the NIHRAC requires notification of adverse events not attributed to the therapy. However, although institutions failing to comply with $\mathrm{NIH}$ could face termination of their federal funding, some researchers are still reluctant to submit findings for public discussion.

Currently investigators are operating in a "gray area," particularly if they conclude on their own that deaths or other adverse events are incidental to the gene therapy procedures being tested, some observers point out. In fact, that grayness is even trickier because the participants in many of these clinical trials are often desperately sick from their underlying disease at the time they agree to take part in the gene transfer procedure.

The long-standing joint undertaking has generated a broader set of issues, including criticism of duplicate regulatory review as well as fears that this duality might even lead to loopholes in jurisdiction. "Dual oversight is unique to gene therapy," says Philip Noguchi, who heads the Division of Cellular and Gene Therapy at the FDA Center for Biologics Evaluation and Research (CBER).

Frequently over the past decade, gene therapy researchers in industry and at universities have raised objections to this dual oversight-with some impact. For instance, the detailed review of proposed clinical trials has shifted from NIHRAC to FDA during the past several years, following a decision by NIH director Harold Varmus to move NIHRAC away from analyzing every proposal, as was once customary.

Nonetheless, NIHRAC continues to consider special cases along the hypothetical frontier of gene therapy, to deliberate over ethical issues, and, through ORDA, to maintain a database describing the more than 300 clinical gene therapy trials and transfer procedures that have been undertaken since 1990 .

In early November, to remind researchers in this field of their continuing responsibilities under this system in which "FDA and NIH each make unique and complementary contributions," CBER Director Kathryn Zoon took a forceful step to close reporting loopholes when she sent a "dear gene therapy IND [investigational new drug] sponsor/principal investigator letter" reviewing requirements for regulatory compliance. It specifies that investigators and sponsors notify both federal agencies of "serious adverse events." At the same time, besides examining the safety of adenovirus gene vectors, NIHRAC members will be considering how to clarify the committee's charter to avoid any lingering ambiguities about reporting responsibilities insofar as safety and adverse events are concerned.

Jeffrey L. Fox 